\title{
Right hemicolectomy with central vascular ligation in colon cancer
}

\author{
Klaus Weber • Werner Hohenberger
}

Published online: 27 July 2011

(C) Springer Science+Business Media, LLC 2011

We read with interest the report by Spasojevic et al. [1] describing the origin of the right colic artery and the implications for oncologic resection of the ascending colon. Using computed tomographic (CT) angiography, the authors demonstrated a right colic artery arising from the superior mesenteric artery in 54\% of patients, with this vessel passing posterior to the superior mesenteric vein in only $7.4 \%$ of cases.

Contrary to the authors' suggestion, we did not fail to report the described variations in our series of extended lymphadenectomy for right colon cancer [2]. Rather, it is our surgical experience that a true right colic artery is rarely seen. Our finding of a true right colic artery in approximately $15 \%$ of patients is consistent with that of previous reports $[3,4]$.

Perhaps the discrepancy between our experience and that of Spasojevic et al. [1] is partly a matter of definition. We and others have observed, for example, that the middle colic artery has many variations. VanDamme and Bonte [5] described two or more middle colic arteries with separate origins in $25 \%$ of their patients. When performing a complete mesocolic excision for right-sided colon cancer, we do not routinely divide such middle colic vessels centrally because lymph node metastases are not seen in this distribution. When, however, a true right colic artery originating from the superior mesenteric artery and passing posterior to the superior mesenteric vein is identified, then a central ligation should be performed. By rotating the fully mobilized colon in a clockwise direction, the origin of both the right colic artery (when present) and the ileocolic artery

K. Weber $(\bowtie) \cdot$ W. Hohenberger

Department of Surgery, University Hospital, Krankenhausstrasse

12, 91054 Erlangen, Germany

e-mail: klaus.weber@uk-erlangen.de may be fully exposed to allow true central ligation [2]. Thus, an arterial stump with potentially involved lymph nodes is never missed. In the procedure, the autonomic nerve plexus enveloping the superior mesenteric artery must be preserved to avoid postoperative diarrhea.

As correctly observed by Spasojevic et al. [1], the right mesocolon is not clearly defined at its medial aspect. When a complete mesocolic excision for right-sided colon cancer is performed, the fascia on both sides of the mesocolon, including the contained ileocolic artery (and the right colic artery when present) must be actively divided in a plane parallel to the mesenteric root. In this way the excision of the mesocolon and its mesocolic lymph nodes is maximized.

Disclosures Klaus Weber and Werner Hohenberger have no conflicts of interest or financial ties to disclosure.

\section{References}

1. Spasojevic M, Stimec BV, Fasel JF, Terraz S, Ignjatovic D (2011) $3 \mathrm{D}$ relations between right colon arteries and the superior mesenteric vein: a preliminary study with multidetector computed tomography. Surg Endosc 25:1883-1886

2. Hohenberger W, Weber K, Matzel K, Papadopoulos T, Merkel S (2009) Standardized surgery for colonic cancer: complete mesocolic excision and central ligation: technical notes and outcome. Colorect Dis 11:354-365

3. Van Damme J-P, Bonte J (1990) The more exceptional right colic artery. In: Van Damme J-P, Bonte J (eds) Vascular anatomy in abdominal surgery. Thieme, Stuttgart

4. Garcia-Ruiz A, Milsom JW, Ludwig KA, Marchesa P (1996) Right colonic arterial anatomy: implications for laparoscopic surgery. Dis Colon Rectum 39:906-911

5. Van Damme J-P, Bonte J (1990) The composed system of the middle colic artery. In: Van Damme J-P, Bonte J (eds) Vascular anatomy in abdominal surgery. Thieme, Stuttgart 